\title{
UTILIZAÇÃO DA PIPER METHYSTICUM (L.) E PASSIFLORA INCARNATA (L.) NO TRATAMENTO DE TRANSTORNO DE ANSIEDADE GENERALIZADA
}

\author{
USE OF PIPER METHYSTICUM (L.) AND PASSIFLORA INCARNATA (L.) IN THE \\ TREATMENT OF GENERALIZED ANXIETY DISORDER
}

\author{
Manuela Conceição da Silva ${ }^{1}$ \\ Natalia Batista de Souza ${ }^{2}$ \\ Thifani dos Santos Rocha ${ }^{3}$ \\ Juliana Azevedo da Paixão ${ }^{4}$
}

Ana Maura Carvalho Moura de Alcantara ${ }^{5}$

\begin{abstract}
RESUMO: As medidas de controle recomendadas no contexto da pandemia do COVID-19 tem afetado a população em muitos calibres das condições de vida e de saúde e, entre elas, de forma significativa o componente de saúde mental como a ansiedade e a depressão. Objetivo: Revisar, através da literatura científica, a eficácia, interações farmacológicas e benefícios do uso dos fitoterápicos kava-kava (Piper methysticum L.) e maracujá (Passiflora incarnata L.) como alternativas terapêuticas para o tratamento do Transtorno de Ansiedade Generalizada (TAG), agravados em tempos de pandemia. Métodos: Trata-se de uma revisão bibliográfica sistemática qualitativa, na qual utilizou-se os sites eletrônicos SciELO, PubMed, Google acadêmico, ANVISA e OMS para a busca de dados. Resultados: Observou-se que os principais benefícios da utilização de fitoterápicos diz respeito ao custo reduzido, à facilidade de acesso e ao baixo índice de efeitos colaterais e reações adversas. Encontrou-se um número considerável de informações que demonstraram a utilização e a procura de fitoterápicos à base de $P$. Incarnata para tratar as psicopatologias como o TAG,
\end{abstract}

\footnotetext{
${ }^{1}$ Acadêmica do curso de farmácia da Universidade Salvador (UNIFACS), Salvador - BA. E-mail:manusilva9ı@@gmail.com.

${ }^{2}$ Acadêmica do curso de farmácia da Universidade Salvador (UNIFACS), Salvador - BA. E-mail: natybior7@gmail.com.

${ }^{3}$ Acadêmica do curso de farmácia da Universidade Salvador (UNIFACS), Salvador - BA. E-mail: thifani.rocha@outlook.com.

${ }^{4}$ Farmacêutica pela Universidade Federal da Bahia (UFBA); especialista em farmacologia aplicada à prática clínica pela AVM-Faculdade Integrada; mestre em recursos genéticos vegetais pela Universidade Estadual de Feira de Santana (UEFS); docente na Universidade Salvador (UNIFACS), Salvador-BA.E-mail: juliana.paixao@unifacs.br.

${ }^{5}$ Farmacêutica e especialista em farmácia clínica e hospitalar pela Faculdade de Tecnologia e Ciências (FTC), Salvador - BA. E-mail: anamauracarvalho@gmail.com.
} 
durante o isolamento social e a quarentena. Não foram encontrados números significativos de estudos capazes de comprovar a utilização da $P$. Methysticum durante a pandemia da COVID-19. Considerações finais: Sugere-se que as espécies P. Incarnata e P. Methysticum são eficazes para o tratamento do TAG por possuir alto valor medicinal com propriedades farmacológicas ansiolíticas, anticonvulsivantes e sedativas.

Palavras-chave: TAG. Fitoterápicos. Covid-ı. Piper methysticum (L.). Passiflora incarnata (L.).

ABSTRACT: The control measures recommended in the context of the COVID-I9 pandemic have affected the population in many calibers of living and health conditions, and among them significantly the mental health component such as anxiety and depression. Objective: To review, through scientific literature, the efficacy, pharmacological interactions and benefits of using the herbal medicines kava-kava (Piper methysticum L.) and passion fruit (Passiflora incarnata L.) as therapeutic alternatives for the treatment of Generalized Anxiety Disorder (GAD), aggravated in times of pandemic. Methods: This is a qualitative systematic literature review, which used the electronic sites SciELO, PubMed, Google Scholar, NHSA and WHO to search for data. Results: It was observed that the main benefits of the use of herbal medicines are the reduced cost, easy access, and low rate of side effects and adverse reactions. A considerable number of information was found that demonstrated the use and demand for P. Incarnata-based herbal medicines to treat psychopathologies such as GAD, during social isolation and quarantine. No significant numbers of studies were found that could prove the use of $\mathrm{P}$. Methysticum during the COVID-I9 pandemic. Final remarks: It is suggested that P. Incarnata and P. Methysticum species are effective for the treatment of GAD by having high medicinal value with anxiolytic, anticonvulsant and sedative pharmacological properties.

Key words: GAD. Phytotherapeutics. Covid-19. Piper methysticum (L.). Passiflora incarnata (L.).

\section{INTRODUÇÃO}

Diante da pandemia do COVID-ı, pessoas que precisaram acatar a quarentena apresentaram maior prevalência de depressão e ansiedade, podendo chegar às situações drásticas, como o suicídio (Mara Pereira et al., 2020; OMS, 2017). A Organização PanAmericana de Saúde (OPAS) (2020), calculou que existe três vezes mais a dominância de sintomas de ansiedade e depressão em vários países, incluindo o Brasil. De acordo com o contexto pandêmico, o medo de contágio, o isolamento social e a perda de membros familiares são agravantes para escassez da saúde mental (OPAS, 2020).

As medidas de controle recomendadas no contexto da pandemia do COVID-I9 tem afetado a população em muitos calibres das condições de vida e de saúde e, entre elas, de forma significativa o componente de saúde mental como a ansiedade e a depressão. Indivíduos com psicopatologia tendem a apresentar níveis mais elevados de estresse e sofrimento psicológico durante a quarentena provocada pela COVID-19, comparados a pessoas sem esse diagnóstico (Mara Pereira et al., 2020). 
Houve um aumento de casos de transtorno de ansiedade entre indivíduos de todo o mundo. Estudos apontam o Transtorno de Ansiedade Generalizada (TAG) como um dos distúrbios psiquiátricos mais comuns entre os transtornos de ansiedade (Lucas Oliveira et al., 2020). A OMS (2017) mostra dados sobre a prevalência de portadores do TAG, onde 3,6\% da população mundial representa as pessoas ansiosas, trazendo esse dado para a população brasileira o número aumenta para 9,3\% (OMS, 20I7).

De um modo geral, observa-se um crescimento na utilização de medicamentos, onde grande parte da população brasileira recorre ao uso de plantas medicinais e fitoterápicos como uma alternativa em relação à utilização de medicamentos sintéticos, além dos fitoterápicos e plantas serem de fácil acesso (Andrade Oliveira et al., 2017). Os fitoterápicos representam tratamentos de menor valor econômico, e se forem utilizados corretamente podem causar menor risco de reações adversas e dependência química, e estão inseridos nas diretrizes e políticas da fitoterapia, sendo empregados no tratamento da ansiedade e de outras doenças (Lucas Oliveira et al., 2020).

Pesquisas realizadas mostraram que para o tratamento de ansiedade, diversas plantas medicinais e fitoterápicos que constam na Instrução Normativa $\mathrm{n}^{\mathrm{o}}$ 02/2014, podem ser consumidas, tais como, a kava-kava (Piper methysticum), maracujá (Passiflora incarnata), valeriana (Valeriana officinalis), camomila (Matricaria recutita), erva-cidreira (Melissa officinalis) e o mulungu (Erythrina mulungu) que são espécies com números elevados de estudos envolvendo pacientes com transtorno de ansiedade generalizada (TAG) (MAINGREDY et al., 2016).

No entanto, presente artigo destacam-se a kava-kava (Piper methysticum) e o maracujá (Passiflora incarnata), por se tratarem de plantas que constituem componentes químicos que apresenta ação ansiolítica, induzindo ao relaxamento e ao sono mostrando resultados mais eficazes para a saúde no controle das crises de ansiedade, por conta da presença de seus princípios ativos fitoterápicos que beneficiam a eficácia farmacológica (BARBOSA et al., 2013; Lucas Oliveira et al., 2020).

Considerando a Kava-kava ( $P$. methysticum) e o maracujá ( $P$. incarnata) serem possíveis opções fitoterapêuticas para o tratamento do TAG agravados em processos de pandemia, o presente trabalho tem como objetivo revisar, através da literatura científica, a eficácia, interações farmacológicas e benefícios do uso dos fitoterápicos kava-kava (Piper methysticum L.) e maracujá (Passiflora incarnata L.) como alternativas terapêuticas para o tratamento do TAG, agravados em tempos de pandemia.

O cenário da pandemia elevou os casos de ansiedade e depressão provocando um grave problema de saúde pública e com isso faz-se necessário traçar estratégias para o cuidado e tratamento dessas doenças. A terapia com plantas, tem sido um método eficaz, de baixo custo que já vem sendo utilizado como opção nesse tipo de tratamento. Deste modo, o presente estudo colabora de maneira substancial trazendo a passiflora e a kavakava como opção fitoterápica.

\section{MÉTODOS}


Trata-se de uma revisão bibliográfica sistemática qualitativa sobre a utilização dessas plantas medicinais, os efeitos colaterais provenientes dessa terapia e a economia no uso destas plantas, tendo em vista o aumento do número de casos provocados pelo advento da Pandemia da COVID-ı9 no Brasil.

As seguintes bases de dados foram utilizadas para pesquisa: Scientific Electronic Library Online (SciELO), National Library of Medicine (PubMed), Google acadêmico, Agência Nacional de Vigilância Sanitária (ANVISA) e Organização Mundial de Saúde (OMS). Foram utilizados os descritores "Piper methysticum", "Passiflora incarnata", combinados com "transtorno de ansiedade generalizada" ou "plantas medicinais ou fitoterapia" ou "covid-I9" ou "interações farmacológicas", incluindo operadores como "AND" e "OR".

Dentre os critérios de inclusão foram selecionados artigos com estudos clínicos e pré-clínicos, e revisões de literatura que relatassem a eficácia das plantas medicinais selecionadas no presente trabalho e pesquisas de campo que estivessem entre o período de 2000 a 202I. Foram optados por artigos científicos escritos nos idiomas português e inglês. Já os critérios de exclusão foram artigos que não possuem o título compatível com as palavras-chave pesquisadas, que não estivessem nos idiomas escolhidos, não indexados nas bases de dados.

Foram encontrados ao todo 3.316 artigos, destes, 53 foram selecionados para leitura e 29 para a elaboração do artigo. Os demais foram excluídos por não se encaixarem nos critérios de inclusão. A seguir, é exposto na figura I, como procedeu-se a realização de pesquisa e busca de artigos.

Figura r: Prospecção da Pesquisa e Busca de Artigos.

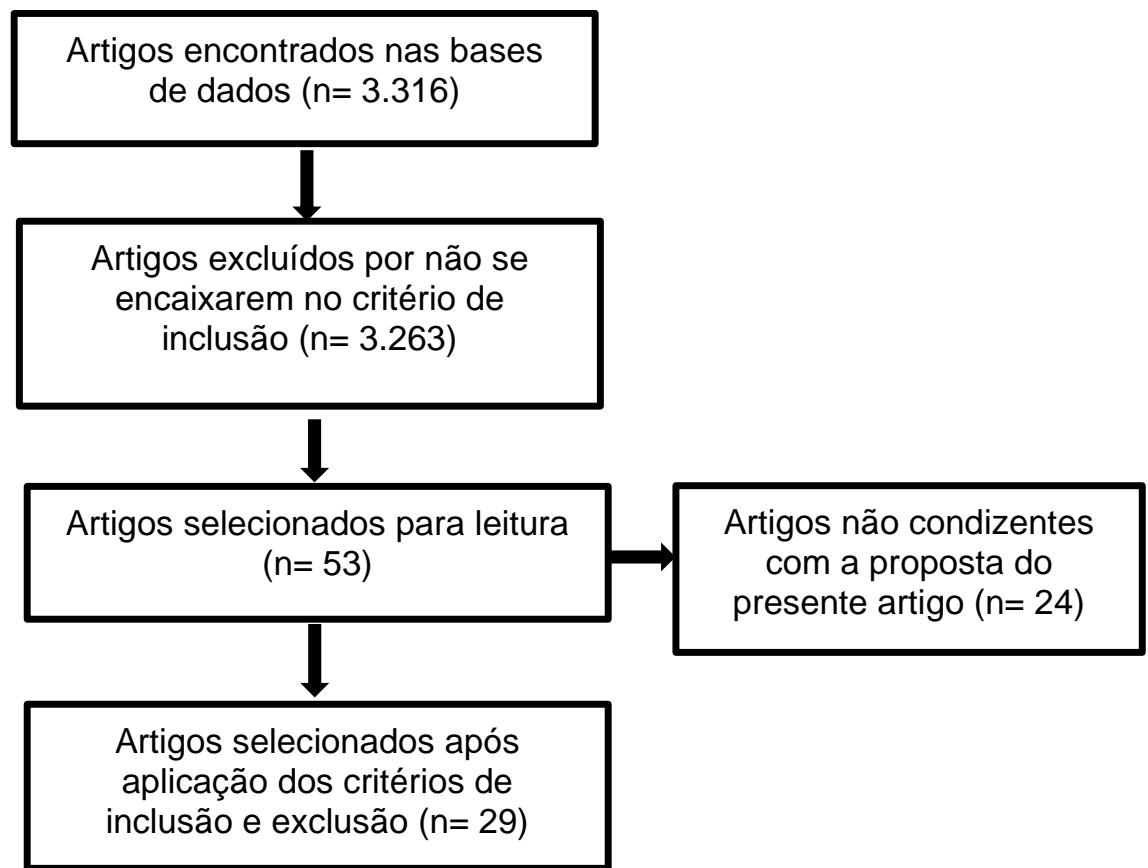

Fonte: Autores (2021). 


\section{RESULTADOS}

\section{POTENCIAL DO USO DA KAVA-KAVA E MARACUJÁ NO TRANSTORNO DE ANSIEDADE GENERALIZADA (TAG)}

De acordo com Zuardi (2017), uma das principais características do TAG é a preocupação excessiva e persistente, onde os sintomas físicos como por exemplo taquicardia, inquietação, insônia, fadiga, dores musculares, dificuldade de relaxar, sudorese, dificuldade de concentração, se cansar facilmente e a preocupação afeta de maneira significativa o desempenho do indivíduo durante as atividades do cotidiano. Por ser uma das doenças mais subdiagnosticadas, a maioria das pessoas que sofrem com esse transtorno procuram um profissional de saúde especializado em saúde mental. $\mathrm{O}$ autor ainda reitera que, para um diagnóstico preciso, há uma necessidade que o paciente apresente de um a três dos sintomas físicos durante seis meses na maioria dos dias (ZUARDI, 20I7).

Duan L e Zhu G (2020), asseguram que durante a pandemia do covid-I9 houve um aumento significativo de transtornos psicológicos, incluindo ansiedade, estresse e depressão. Atrelado a isso, o isolamento social é um grande fator para o aumento de efeitos negativos na saúde mental da população (Carlos Lima et al., 2017). Nesse contexto, para quadros de ansiedade a Piper méthysticum e a Passiflora incarnata são fitoterápicos indicados como tratamento alternativo e de baixo custo o que, facilita a adesão ao tratamento (CORDEIRO et al., 2005).

\section{Piper methysticum G. Frost}

Originada nas ilhas do Pacífico Sul, a Piper methysticum, família Piperaceae, é utilizada há anos para promover bem-estar, redução da fadiga e da ansiedade. Atualmente tem sido utilizada como tratamento alternativo para distúrbios psiquiátricos, como o transtorno de ansiedade generalizada (TAG) que tem sofrido um aumento significativo durante a pandemia do covid-ı9 (DUAN e ZHU, 2020; PERES et al., 2014).

O rizoma da $P$. methysticum em forma de extrato seco padronizado, são as partes mais utilizada para fins fitoterapêuticos, onde encontra-se os princípios ativos mais relevantes como as cavapironas (também chamadas de kavalactonas) e flavonoides. A atividade simultânea dessas substâncias proporciona relaxamento muscular, ação antiarrítmica, redução no estresse e na ansiedade. (BARBOSA et al., 2013).

De acordo com Justo e Silva (2008), a P. methysticum possui uma boa eficácia e ação farmacológica no sistema nervoso central (SNC). Isso acontece em decorrência da ação antagonista das kavalactonas, onde as seis principais são yagonina (YAN), desmetoxiagonina (DXY), kavaína (KAV), dihidrokavaína (DHK), metisticina (MET) e dihidrometisticina (DHM), que se ligam aos receptores do ácido gama-aminobutírico (GABA), e possivelmente aos receptores $\mathrm{N}$-metil-D-aspartato (NMDA) e/ou canais de sódio dependente de voltagem e inibem a captação de noradrenalina, possibilitando atividades ansiolíticas, anticonvulsivantes, sedativas, espasmolíticas e analgésicas. (JUSTO e SILVA, 2008). 
As kavalactonas tem o poder inibitório de várias isoformas do CYP45o, e essa virtude causa inúmeras interações, principalmente farmacocinéticas, com outras drogas, pois reduz a metabolização destas pelas enzimas inibidas do complexo $\mathrm{CYP}_{450}$, podendo induzir a toxicidade (JUSTO e SILVA, 2008). As vantagens da utilização deste fitoterápico comparado aos medicamentos sintéticos é a boa tolerabilidade e a ação sem gerar sonolência, causando assim um fácil desmame sem provocar dependência medicamentosa quando utilizado em dose diária de 60-210 mg por no máximo três meses (PERES et al., 2014; BRASIL, 2016).

A P. methysticum quando utilizada em doses recomendadas não provoca efeitos adversos, porém, há relatos que demonstram que o uso prolongado desse fitoterápico pode causar reações alérgicas, queixas gastrointestinais, cefaleia, tonturas, pigmentação da pele, fadiga matinal (logo no início do tratamento), movimentos involuntários, detrimentos de deglutição e respiração, contrações involuntárias não ritmadas e contínuas nas extremidades, perda de peso, perda da função hepática e renal. Caso apresente alguns desses efeitos deve-se suspender a utilização imediatamente (PERES et al., 20I4; BRASIL, 2016).

Pacientes com problemas hepáticos, possivelmente, não possam fazer o uso desse fitoterápico. Estudos mostram que houve diversos casos de hepatotoxicidade no Brasil, onde ocorreu lesão hepática que ocasionou transplantes hepáticos e óbitos. Países como a Suíça e a Alemanha suspenderam o uso desse fitoterápico, pois ocorreram vários casos de toxicidade hepática grave (hepatite, cirrose e falência hepática) que foram relacionados com o uso do extrato desta espécie (BARBOSA et al., 2013).

Em relação ao uso dessa planta medicinal em mulheres que amamentam, deve-se ser abdicada o uso, pois constituintes presentes na $P$. methysticum podem passar para o leite materno e estimulam a perda do tônus uterino, assim, complicando uma futura gravidez (CORDEIRO et al., 2005; JUSTO e SILVA, 2008). Atrelado a isso, nota-se que a $P$. methysticum, quando usado em conjunto com álcool, medicamentos sintéticos que atuam no SNC e outras plantas medicinais (erva-dos-gatos, aipo, camomila alemã, cálamo, calêndula, ênula, valeriana, sassafrás, urtiga, sálvia, ginseng, siberiano, erva-cidreira e erva de são joão) podem potencializar os efeitos farmacológicos e adversos, e aumentar a possibilidade de uma lesão hepática, assim, ocasionando interações farmacológicas (JUSTO e SILVA, 2008).

\section{Passiflora incarnata Linnaeus}

Pertencente à família Passifloraceae e originada na América do Sul e do Norte, a $P$. Incarnata é um vegetal que possui folhas simples, suas flores apresentam-se perfumadas, largas, com pedúnculos esbranquiçados e pétalas de coloração azul clara, roxa, branca e lavanda. Os frutos, de coloração marrom-avermelhada ou verde clara, são arredondados e compostos de casca, polpabranca e sementes revestidas por arilo amarelado (Ana Santos et al., 2020).

A P. incarnata possui alto valor medicinal como antiespasmódico, antiepiléptico, antiasmático, narcótico, anti-histérico, ansiolítico e sedativo, em decorrência da presença de compostos bioativos passiflorina, flavonoides, alcaloides e c-glicosideos utilizados no desenvolvimento de medicamentos auxiliares para o tratamento da ansiedade com atividade sedativa (Juliane Pessolato et al., 202I). 
Seu mecanismo de ação é pela inibição da monoamina oxidase (MAO) e ao mesmo tempo, ativa os receptores GABA, com afinidade para os receptores GABA (A) e GABA (B). A atividade GABAérgica acontece através de neurônios que secretam o GABA com a ligação dos receptores GABA (A). Ações que a P. Incarnata induz no SNC promovendo os efeitos farmacológicos da substância (Lucas Oliveira et al., 2020).

Os flavonoides (orientina, isoorientina, isovitexina e vitexina) encontrados no vegetal são os principais responsáveis pelas atividades farmacológicas. Junto com os alcaloides (harmina, harmana, harmol, harmolol e harmalina) também presentes, desenvolvem ações depressoras inespecíficas do SNC. Deste modo, o vegetal apresenta propriedades ansiolíticas, anticonvulsionantes e sedativas (Mayke Lopes et al., 2017).

A espécie vegetal ganha destaque quando comparada a outros medicamentos psicotrópicos por possuir menor probabilidade de efeitos colaterais e dependência. Além disso, as reações adversas do uso incluem fadiga, náuseas,vômitos, cólicas e sonolência, podendo também ser observado com certa frequência de cefaleia, febre/mialgia, epigastralgia, insônia, adinamia, dor torácica e agitação (Mayke Lopes et al., 2017; BRASIL, 2016).

As interações importantes ocorrem principalmente com bebidas alcoólicas e outras drogas, aumentando a sonolência quando ingerida com álcool, e estimulando a ação de hipnóticos e ansiolíticos. Pode provocar hemorragias se ingerida em altas doses junto com antiplaquetários e anti-inflamatórios não esteroidais (AINES). Seu uso simultâneo com fármacos neuropsíquicos diminui o fluxo sanguíneo, podendo causar dormência corporal (TORCHI et al., 20I4; ARAÚJO et al., 202I).

O uso da $P$. incarnata com álcool ou outras drogas sedativas-hipnóticas poderá aumentar a intensidade de sonolência de benzodiazepínicos (BZD) como o lorazepam ou Diazepam, barbitúricos como o fenobarbital, narcóticos como a codeína, o uso sinérgico com drogas inibidoras da monoamino oxidase (isocarboxazida, fenelzina e tranilcipromina) poderá causar efeito aditivo. Estudos proprõem que a administração concomitantemente com aspirina, varfarina ou heparina e antiplaquetários como clopidogrel e, ainda, com drogas anti-inflamatórias não esteroidais como ibuprofeno e naproxeno podem causar sangramento. A utilização dessa planta com cafeína, guaraná ou efedra poderá causar aumento da pressão arterial (ARAÚJO et al., 2021; Fabiana Ferreira, 2019).

\section{UTILIZAÇÃO DA PIPER METHYSTICUM E PASSIFLORA INCARNATA NO TRANSTONO DE ANSIEDADE GENERALIZADA (TAG) DURANTE A PANDEMIA DO COVID-19}

Os artigos selecionados como amostra no Quadro I, foram associados de acordo com os autores, ano, revista publicada e principais achados. A partir da avaliação, apenas 3 artigos discorrem sobre estudo de caso realizado com pessoas que estão fazendo o uso de plantas medicinais, principalmente a base de P. incarnata durante a pandemia do covidI9, os demais são artigos citam o aumento do uso dessa planta durante a pandemia. 
Quadro r: Síntese dos Principais Achados sobre o uso da P. incarnata durante a pandemia da covid-I9.

\begin{tabular}{|c|c|c|c|c|}
\hline $\mathbf{N}$ & Ano & Autores & Revista & Principais Achados \\
\hline I & $202 \mathrm{I}$ & $\begin{array}{c}\text { ABREU LPS e } \\
\text { MARTINAZZO } \\
\text { AP }\end{array}$ & $\begin{array}{c}\text { Brazilian } \\
\text { Journal of } \\
\text { Development }\end{array}$ & $\begin{array}{l}\text { A busca pelo uso de } \\
\text { produtos naturais na } \\
\text { prevenção de infecção por } \\
\text { Covid-ı́; } \text { Passiflora } \\
\text { incarnata é uma das } \\
\text { plantas medicinais mais } \\
\text { utilizadas e citadas para } \\
\text { aliviar os sintomas } \\
\text { causados pela pandemia e } \\
\text { na prevenção de infecção } \\
\text { por covid-ı. }\end{array}$ \\
\hline 2 & 2020 & $\begin{array}{l}\text { Conselho Federal de } \\
\text { Farmácia (CFF) }\end{array}$ & $\begin{array}{l}\text { Conselho } \\
\text { Federal de } \\
\text { Farmácia }\end{array}$ & $\begin{array}{l}\text { Venda de medicamentos } \\
\text { psiquiátricos cresce na } \\
\text { pandemia; Aumento de } \\
59 \% \text { de fitoterápicos a } \\
\text { base de Passiflora } \\
\text { incarnata comprado ao } \\
\text { ano de 20I9. }\end{array}$ \\
\hline 3 & 2021 & $\begin{array}{c}\text { PESSOLATO JP et } \\
\text { al. (2021) }\end{array}$ & $\begin{array}{c}\text { Brazilian } \\
\text { Journal of } \\
\text { Health Review }\end{array}$ & $\begin{array}{l}\text { Avaliação do consumo de } \\
\text { Valeriana e Passiflora } \\
\text { durante pandemia } \\
\text { COVID-ı; Aumento no } \\
\text { consumo de Passiflora } \\
\text { Incarnata na pandemia do } \\
\text { novo Corona Vírus em } \\
2020 \text {, comparado ao } \\
\text { mesmo período de } 2019 .\end{array}$ \\
\hline 4 & 2020 & $\begin{array}{c}\text { SHAHRAJABIAN, } \\
\text { M. }\end{array}$ & $\begin{array}{l}\text { Phytotherapy } \\
\text { Research }\end{array}$ & $\begin{array}{l}\text { Medicamentos } \\
\text { tradicionais à base de } \\
\text { ervas para superar o } \\
\text { stress, ansiedade e } \\
\text { melhorar a saúde mental } \\
\text { em surtos de coronavírus } \\
\text { humanos; A Passiflora } \\
\text { Incarnata utilizada nos } \\
\text { sintomas ansiedade }\end{array}$ \\
\hline
\end{tabular}




\begin{tabular}{|c|c|c|c|l|}
\hline & & & $\begin{array}{l}\text { ocasionados pela } \\
\text { pandemia do Corona } \\
\text { Vírus. }\end{array}$ \\
\hline 5 & 2020 & $\begin{array}{c}\text { SILVA ALS et al. } \\
(2020)\end{array}$ & $\begin{array}{l}\text { Brazilian Journal of } \\
\text { Natural Sciences }\end{array}$ & $\begin{array}{l}\text { Uso de plantas medicinais no } \\
\text { tratamento de ansiedade no } \\
\text { ambiente acadêmico; uso de } \\
\text { plantas medicinais incluindo } \\
\text { a Passiflora incarnata no } \\
\text { tratamento do TAG que } \\
\text { sofreu um aumento durante } \\
\text { o momento pandêmico. }\end{array}$ \\
\hline
\end{tabular}

Fonte: Autores (202I).

Diante de dados levantados pelo Conselho Federal de Farmácia (CFF), e uma reportagem produzida pelo Jornal Estado de Minas, onde o tema foi os efeitos da pandemia de Covid-ig sobre a saúde mental dos brasileiros, houve um aumento do consumo de fitoterápicos que aliviam sintomas de ansiedade e insônia. Dados apresentados pela subsidiária brasileira da farmacêutica Aspen Pharma demonstra que em maio de 2019 a demanda por um dos fitoterápicos que comercializa entre os dois que produz à base de $P$. Incarnata, era de 118 mil unidades, chegando agora a 188 mil vendidas, um aumento de $59 \%$ $(C F F, 2020)$.

Com a pandemia do COVID-ı9 no ano de 2020, a fim de proporcionar a diminuição da propagação do vírus e seu contágio, uma das medidas tomadas foi o isolamento social e a quarentena impossibilitando a população de realizar as atividades de rotina, ditas "normais". Essas mudanças repentinas no estilo de vida das pessoas desencadeou o desenvolvimento de transtornos de ansiedade, insônia e stress e entre outros sintomas. Sem acesso a consulta com profissionais devidamente capacitados para a indicação e prescrição de medicamentos alopáticos para tratamentos, ocorreu um grande aumento na procura por ansiolíticos naturais de fácil acesso em farmácias, de modo a diminuir esses sintomas, pelo fato de os produtos serem naturais (Juliane pessolato et al., 202I).

A Passiflora incarnata foi um dos produtos naturais mais procurados, durante a pandemia para tratamento auxiliar de crises de ansiedade, por ser um medicamento associado ao conhecimento popular como ansiolítico que desempenha a ação calmante, podendo ajudar as pessoas durante e após esse período de pandemia, além de ser facilmente encontrado, e tem um custo acessível à população (DA SILVA et al., 2020).

Segundo Shahrajabian et al., (2020) a P. Incarnata foi uma das plantas citadas que pode diminuir significativamente os sintomas da TAG durante a pandemia, com isso vem ocorrendo um grande aumento na comercialização dos fitoterápicos, ganhando a preferência da população com consumo seguro, qualidade e eficácia comprovada através de estudos científicos farmacológicos e farmacoterapêuticos e isento de prescrição médica como consta na Instrução Normativa - IN io/2014 (MINISTÉRIO DA SAÚDE, 2014). 
Um estudo realizado por Juliane Pessolato et al., (202I), evidencia uma comparação de vendas de fitoterápicos de uma farmácia comercial dos meses de abril a julho de 2019 à abril a julho de 2020, que houve, ao todo, um aumento de 523,2\%. Observa-se através desse estudo que o número de vendas de medicamentos fitoterápicos, principalmente a base de Passiflora incarnata, foi muito grande nesse momento pandêmico.

Abreu e Martinazzo, (2021), realizaram um estudo, no qual foram entrevistados 529 pessoas, discorreu-se sobre o uso de produtos naturais na prevenção de infecção do covid-19. A maioria das pessoas que participaram da entrevista relataram sentir medo, preocupação, ansiedade, tristeza e impotência, e indicaram produtos medicinais que aliviam a ansiedade. A Passiflora incarnata foi um dos produtos mais citados durante este estudo, por possuir atividade ansiolítica e sedativa leve e ajudar a aliviar os sintomas descritos. (ABREU e MARTINAZZO, 2021).

Diante do cenário pandêmico também houve um aumento do uso da espécie Piper methysticum para ansiedade, estresse e insônia, que são sintomas do transtorno de ansiedade generalizada (TAG). Em um estudo realizado por Stephen Buhner (2020), a P. methysticum foi utilizada para protocolo específico de sintomas para o tratamento da síndrome de CovidI9 e Pós-Coronavírus, como cefaleia, problemas músculo esqueléticos, mialgia, sensibilidade cutânea, palpitações e dores no peito. Porém, não houve um aumento significativo, possivelmente pela toxicidade apresentada nesta planta, principalmente quando utilizada com outras plantas medicinais. Em virtude disso a $P$. methysticum não pode ser comercializada sem prescrição médica, causando assim uma baixa procura, por não ser de fácil acesso para população (OLADIMEJI E VALAN 202I; Stephen Buhner, 2020; INSTRUÇÃO NORMATIVA, 20I4).

\section{DISCUSSÃO}

A fitoterapia é de grande importância social, por se tratar de uma prática secular da civilização humana que utiliza-se de elementos encontrados em abundância na natureza, onde o conhecimento é passado de geração a geração. Sendo assim, o uso dessa medicina tradicional pela população brasileira tem aumentado no decorrer dos anos, uma vez que, com o avanço da ciência é possível desenvolver fitoterápicos mais seguros e eficazes, de tal modo que estão sendo cada vez mais utilizados como tratamentos alternativos, menos agressivos e mais idôneos para a terapia do TAG. (PERES et al., 20I4).

Os estudos evidenciam que os fitoterápicos representam uma excelente saída para o tratamento de pacientes com um menor poder aquisitivo, visto que muitas famílias foram impactadas financeiramente pela pandemia e que são de grande importância, dentro do contexto da pandemia, devido os agravos de problemas psicológicos em pessoas que já apresentavam algum transtorno, bem como, os altos índices de novos casos dessa patologia.

Sendo assim, os principais benefícios da utilização de fitoterápicos é o custo reduzido, a facilidade de acesso, o baixo índice de efeitos colaterais e reações adversas e a oferta da garantia de qualidade e segurança para os usuários, facilitando a adesão ao tratamento. Além disso, iniciativas como a implementação dos fitoterápicos nas Práticas Integrativas e Complementares em saúde (PICS) no Sistema Único de Saúde (SUS) foi de grande 
importância para a aproximação desse tipo de terapia alternativa aos usuários da atenção primária desse sistema.

Nota-se que a procura por fitoterápicos a base de P. Incarnata durante a pandemia da COVID-Ig teve um aumento significativo, possivelmente por ser de fácil acesso e isenta de prescrição médica, segundo a IN ıo/2014. (Juliane Pessolato et al., 2021; MINISTÉRIO DA SAÚDE, 2014). Apesar da pandemia ser um assunto novo, encontrou-se um número considerável de revisões bibliográficas e pesquisas de campo que demonstrassem a utilização e a procura de fitoterápicos a base de P. Incarnata para tratar as psicopatologias como o TAG, durante o isolamento social e a quarentena.

Entretanto, o mesmo não aconteceu com a $P$. methysticum. Não há números significativos de estudos que comprovem a utilização dessa planta medicinal como fitoterápico durante a pandemia da COVID-19. Atrelado a isso, um dos possíveis motivos para não haver uma grande procura por fitoterápicos à base de desta planta é a comercialização somente com prescrição médica, de acordo com a IN 02/2014 e a ANVISA, por conta dos casos de hepatoxicidade ocorridos no Brasil e em alguns países europeus. (INSTRUÇÃO NORMATIVA, 2014; BARBOSA et al., 2013).

\section{CONSIDERAÇÕES FINAIS}

O desenvolvimento do presente estudo possibilitou uma análise de como a pandemia da COVID-ı9 no Brasil tem refletido na saúde mental da população. Uma reflexão acerca dos transtornos de ansiedade que acomete muitos indivíduos e de como esses números tem crescido mediante o atual cenário mundial de pandemia. Permitiu analisar o uso da passiflora incarnata e piper methysticum, través da fitoterapia como meio alternativo aos tratamentos alopáticos.

De um modo geral, a literatura mostra que a passiflora incarnata e a piper methysticum são eficazes para o tratamento do Transtorno de Ansiedade Generalizada (TAG) por possuir alto valor medicinal com propriedades farmacológicas ansiolíticas, anticonvulsivantes e sedativas e menor probabilidade de efeitos colaterais e dependência.

O estudo trouxe uma abordagem acerca da farmacologia das plantas e a importância da utilização no tratamento da ansiedade, de tal forma, ficou evidente que os objetivos do trabalho foram realmente alcançados.

Os recursos utilizados ajudaram a alcançar os resultados esperados, as pesquisas bibliográficas forneceram informações acerca do atual cenário da pandemia do covid-ı9 no Brasil, sobre os números de portadores de TAG e como a pandemia tem contribuído para o agravo e aumento de novos casos. Detalhou as ações farmacológicas das plantas medicinais analisadas e sua importância como tratamento alternativo dos casos de ansiedade.

Dada a importância do tema, torna-se necessário a continuidade de estudos dessa natureza, visando contribuir para o monitoramento das condições de saúde da população, bem como, na vigilância do uso das substâncias passiflora incarnata e piper 
methysticum, tendo em vista, assegurar o controle sanitário desses fitoterápicos, uma vez que, o uso incorreto pode provocar danos à saúde, e mesmo em baixos níveis, estimular efeitos colaterais importantes.

Nesse sentido a utilização de literatura foi satisfatória e contribuiu de forma enriquecedora mostrando com clareza a importância e eficácia das substâncias passiflora incarnata e piper methysticum no tratamento fitoterápico do Transtorno de Ansiedade Generalizada (TAG).

\section{REFERÊNCIAS}

ABREU, Luciana de Paiva Santos; MARTinAzZO, Ana Paula. A busca pelo uso de produtos naturais na prevenção de infecção por Covid-ı9. Brazilian Journal of Development, v. $7, \quad$ n. $4, \quad$ p. 41613-41650, 2021. Disponível em: https://www.brazilianjournals.com/index.php/BRJD/article/view/28750/22705. Acesso em: I2 de março de 202I.

ARAUJO, Ana carolina Baptista et al. Plantas que agem no Sistema Nervoso Central: O uso dos Fitoterápicos Kava-Kava, Passiflora e Valeriana no tratamento de transtorno de ansiedade. Farmácia na Atenção e Assistência à Saúde. p. I-388-4I6. v.4, 202ı. Disponível em: https://www.atenaeditora.com.br/post-artigo/49854. Acesso em: 2 de março de 202I.

BARBOSA, Diomara Resende; LENARDON, L.; PARTATA, Anette Kelsei. Kava-kava (Piper methysticum): uma revisão geral. Rev. Cien. ITPAC, v. 6, n. 3, p. I-19, 2013. Disponível em: https://assets.unitpac.com.br/arquivos/Revista/63/3.pdf. Acesso em: 26 de janeiro de 2021.

BRASIL. Agência Nacional de Vigilância Sanitária. Memento Fitoterápico - Farmacopeia Brasileira. I $^{\underline{a}}$ edição. Brasília. 20I6. Disponível em: https://www.gov.br/anvisa/pt$\mathrm{br} /$ assuntos/farmacopeia/memento-fitoterapico/memento-fitoterapico.pdf/view. Acesso em: 26 de janeiro de 202I.

BUHNER, Stephen Harrod. SARS-CoV-2 (COVID-I9): HERBAL PROTOCOLS FOR THE TREATMENT OF INFECTION AND POST-CORONAVIRUS SYNDROME. Disponível em: https://www.stephenharrodbuhner.com/wpcontent/uploads/2020/o8/covid-I9-2.update.txt.pdf. Acesso em: I5 de abril de 202I.

CORDEIRO, C. H. G.; CHUNG, M. C.; DO SACRAMENTO, L. V. S. Interações medicamentosas de fitoterápicos e fármacos: Hypericum perforatum e Piper methysticum. Revista Brasileira de Farmacognosia, v. 15, n. 3, p. 272-278, 2005. Disponível em: $\quad$ https://www.scielo.br/scielo.php?pid=Soro2$695 X_{2005000300019} \& s c r i p t=s c i \_a r t t e x t \& t \operatorname{lng}=p t$. Acesso em: 2 de março de 2021.

Conselho Federal de Farmácia (CFF). Venda de medicamentos psiquiátricos cresce na pandemia. Disponível em: <http://covidig.cff.org.br/venda-de-medicamentospsiquiatricos-cresce-na-pandemia/>. Acesso em: 16 abr. 2021; 
DA SILVA, Alana Luisa Sampaio et al. Uso de plantas medicinais no tratamento de ansiedade no ambiente acadêmico. Brazilian Journal of Natural Sciences, v. 3, n. 3, p. 458458, 2020. Disponível em: https://doi.org/Io.31415/bjns.v3i3.124. Acesso em: I5 de março de 2021

DE OLIVEIRA, Lucas Martins; DE MENEZES FILHO, Antonio Carlos Pereira; PORFIRO, Cinthia Alves. Uso da Passiflora incarnata L. no tratamento alternativo do transtorno de ansiedade generalizada. Research, Society and Development, v. 9, n. II, p. e2349119487-e2349119487, 2020. Disponível em: https://rsdjournal.org/index.php/rsd/article/view/9487. Acesso em: 7 março de 202I.

DUAN, L.; ZHU, G. Intervenções psicológicas para pessoas afetadas pela epidemia de COVID-19. The Lancet Psychiatry, v. 7, n. 4, pág. 300-302, 2020. Disponível em: https://doi.org/ro.1016/S2215-0366(20)30073-0. Acesso em: 8 de abril de 2021.

FERREIRA, Fabiana Sari. INTERAÇÕES MEDICAMENTOSAS DE FITOTERÁPICOS UTILIZADOS NO TRATAMENTO DA INSÔNIA: UMA BREVE REVISÃO. Visão Acadêmica, v. 20, n. 3, 2019. Disponível em: https://revistas.ufpr.br/academica/article/view/67826. Acesso em: 8 de abril de 2021.

Instrução normativa no 02 , de 13 de maio de 2014. Publica a "Lista de medicamentos fitoterápicos de registro simplificado" e a "Lista de produtos tradicionais fitoterápicos de registro simplificado". Disponível em: http://bvsms.saude.gov.br/bvs/saudelegis/anvisa/2014/intoo02_13_05_2014.pdf acesso em : I2 de abril de 2021.

JUSTO, Seilin Cardoso; SILVA, Chana Medeiros. Piper methysticum G. Forster (KavaKava): uma abordagem geral. Revista Eletrônica de Farmácia, v. 5, n. I, 2008. Disponível em: https://revistas.ufg.br/REF/article/view/4617. Acesso em: Io de março de 202I

LIMA, Carlos Kennedy Tavares et al. The emotional impact of Coronavirus 2019-nCoV (new Coronavirus disease). Psychiatry research, v. 287, p. II2915, 2020. Disponivel em: https://pubmed.ncbi.nlm.nih.gov/32199182/ acesso em: 20 de abril de 2021.

LOPES, MAYKE WILLIAN; TIYO, ROGERIO; ARANTES, VINICIUS PEREIRA. UTILIZAÇÃO DE PASSIFLORA INCARNATA NO TRATAMENTO DA ANSIEDADE. REVISTA UNINGÁ REVIEW, v. 29, n. 2, 2017. Disponível em: http://revista.uninga.br/index.php/uningareviews/article/view/1952 acesso em: 5 de abril de 202I

MAINGREDY, Xisto et al. FITOTERÁPICOS NO TRATAMENTO DE TRANSTORNOS DA ANSIEDADE. Revista Eletrônica de Farmácia, v. 13, n. I.I, 2016. Disponível em: https://revistas.ufg.br/REF/article/view/4078o acesso em: I de março de 2021

MINISTÉRIO DA SAÚDE. Instrução Normativa n. Io, DE 26 DE NOVEMBRO DE 2014. Disponível 
http://bvsms.saude.gov.br/bvs/saudelegis/anvisa/2014/intooro_26_II_20I4.html. Acesso em 24 abr. 2021;

OLIVEIRA, Andrade, et al., AVALIAÇÃO DO CONHECIMENTO ETNOBOTÂNICO E POPULAR SOBRE O USO DE PLANTAS MEDICINAIS JUNTO A ALUNOS DE GRADUAÇÃO. Revista Brasileira de Gestão Ambiental (Pombal-PB-Brasil), v. II, n. I, p. 07-I3, 2017. Disponível em: https://www.gvaa.com.br/revista/index.php/RBGA/article/view/470r acesso em: ro de março de 2021

OLADIMEJI, Ashaolu Victoria; VALAN, MF. Medicina alternativa à base de fitoterápicos natural para combater a depressão em meio à pandemia covid-I9. 2021.

ORGANIZAÇÃO PAN-AMERICANA DE SAÚDE (OPAS). ONU destaca a necessidade urgente de aumentar investimentos em serviços de saúde mental durante a pandemia de COVID-ig. Dispovivel em: https://www.un.org/sites/un2.un.org/files/un_policy_briefcovid_and_mental_health_final.pdf. Acesso em: 16 abr.2021;

ORGANIZAÇÃO MUNDIAL DE SAÚDE - OMS. Aumenta o número de pessoas com depressão no mundo. 23 de fevereiro de 2017. Disponível em: https://www.paho.org/pt/search/r?keys=aumenta+o+numero+de+pessoas+com+depressa o+no+mundo+Brasil. Acesso em: o9 de abril de 2021.

Organização Mundial de Saúde OMS. Depression and other common mentaldisorders: global health estimates [Internet]. Geneva: WHO; 2017 ${ }^{2}$ Disponível em: http://apps.who.int/iris/bitstream/ro665/254610/I/WHO-MSD-MER-2017.2- eng.pdf Acesso em: o9 de abr de 202i;

PEREIRA, Mara Dantas et al. A pandemia de COVID-i9, o isolamento social, consequências na saúde mental e estratégias de enfrentamento: uma revisão integrativa. Research, Society and Development, v. 9, n. 7, p. e652974548- e652974548, 2020;

PERES, D. M.; PESSUTO, M. B.; LOPES, G. C. Valor Terapêutico de Piper Methysticum: Considerações Gerais e Segurança no Tratamento do Transtorno de Ansiedade Generalizada. Braz. J. Surg. Clin. Res, v. 8, n. 2, p. 83-7, 2014. Disponível em: https://www.mastereditora.com.br/periodico/201410oI_07440I.pdf acesso em: 17 de fevereiro de 2021.

PESSOLATO, Juliane Paula et al. Avaliação do consumo de Valeriana e Passiflora durante pandemia COVID-19. Brazilian Journal of Health Review, v. 4, n. 2, p. 5589-5609, 2021. Disponível em: https://www.brazilianjournals.com/index.php/BJHR/article/view/26397\#: :text=Durant e\%200\%20per\%C3\%ADodo\%2opesquisado\%2C\%2ofoi,durante\%20a\%2opandemia\%20CO VID\%2Di9. Acesso em: is de março de 202I.

SANTOS, Ana Paula Medeiros; GALINDO, Arle Santos; DE SOUZA QUEIROZ, Evandro. Propriedades neuropsicofarmacológicas, compostos quimicamente ativos e uso 
medicinal da passiflora incarnata. Brazilian Journal of Development, v. 6, n. 12, p. 9482394836, 2020. Disponivel em: https://www.brazilianjournals.com/index.php/BRJD/article/view/21060\#: :text=A\%2oP assiflora\%2oincarnata\%20L.\%2C\%2oconhecida,o\%2oextrato\%2onatural\%2oda\%2oP. acesso em: 20 de março de 202I.

SHAHRAJABIAN, Mohamad H. et al. Traditional herbal medicines to overcome stress, anxiety and improve mental health in outbreaks of human coronaviruses. Phytotherapy Research, 2020. Disponível em: https://onlinelibrary.wiley.com/doi/ro.1002/ptr.6888 acesso em: I9 de abril de 202I.

TORCHI, Camila et al. Seguimento do uso da Passiflora Incarnata no tratamento da insônia. II Simpósio de assistência farmacêutica. Disponível em: http://www.saocamilosp.br/novo/eventos-noticias/saf/resumo-27.pdf Acesso em: 22 de abril de 202I v. 29.

ZUARDI, A. W. Características básicas do transtorno de ansiedade generalizada. Medicina (Ribeirão Preto), [S. l.], v. 50, n. supl.I, p. 51-55, 2017. DOI: 10.11606/issn.21767262.v5oisupli.p5I-55. Disponível em: https://www.revistas.usp.br/rmrp/article/view/I27538 Acesso em: I7 de fevereiro de 202I. 